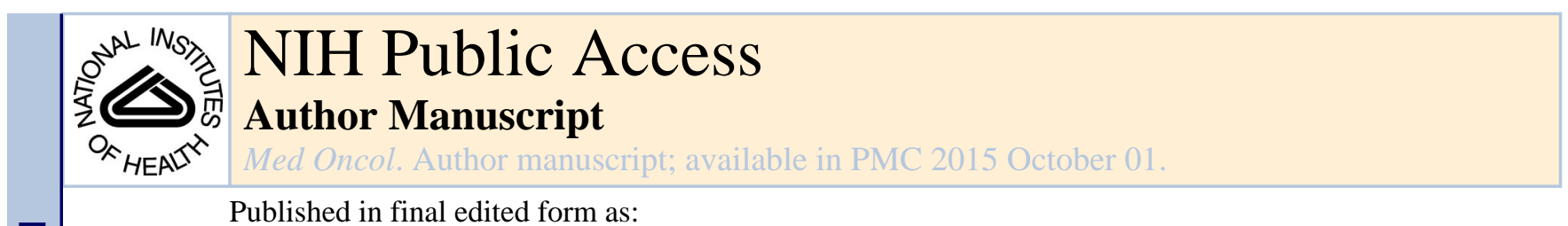

Published in final edited form as:

Med Oncol. 2014 October ; 31(10): 187. doi:10.1007/s12032-014-0187-1.

\title{
Extrapulmonary small cell carcinoma: the University of Kansas experience and review of literature
}

\author{
Christopher S. R. Dakhil, \\ Cancer Center of Kansas, Wichita, KS, USA \\ Jo A. Wick, \\ Department of Biostatistics, University of Kansas Medical Center, Kansas City, KS, USA \\ Anup Kasi Loknath Kumar, \\ Division of Hematology and Oncology, University of Kansas Medical Center, Kansas City, KS, \\ USA
}

\begin{abstract}
Megha Teeka Satyan, and
Division of Hematology and Oncology, University of Kansas Medical Center, Kansas City, KS, USA

Prakash Neupane

Division of Hematology and Oncology, University of Kansas Medical Center, Kansas City, KS, USA
\end{abstract}

Christopher S. R. Dakhil: cdakhil@gmail.com; Jo A. Wick: jwick@kumc.edu; Anup Kasi Loknath Kumar: akasi@kumc.edu; Megha Teeka Satyan: meghaanup19@gmail.com; Prakash Neupane: pneupane@kumc.edu

\section{Abstract}

Though extrapulmonary small cell carcinoma was first described over 80 years ago, definitive treatment recommendations are lacking. The treatment strategies commonly utilized are extrapolated from pulmonary small cell carcinoma experience. A better understanding of this entity is needed to improve management approach. The University of Kansas tumor registry was reviewed from 1990 to 2013. Thirty-five cases met the inclusion and exclusion criteria for review. Age, gender, smoking status, weight loss, metastatic disease-related data, stage, performance status (PS), treatment received, and survival data were collected. Patients were evaluated with a variety of primary locations of disease including GI tract (29\%), GU tract (35\%), Gyn organs (17 $\%)$, head and neck (14\%), and unknown primary (9\%). Several sites of metastatic disease were noted, with 57 and $43 \%$ of patients meeting criteria for limited disease (LD) and extensive disease (ED), respectively. Chemotherapy, surgery, and radiation were used in several different regimens, with small cell lung cancer-type regimens incorporating a platinum and etoposide being the most common (74\%). Patients with LD had a median survival of 36 months compared with 5 months in patients with $\mathrm{ED}(p<0.0001)$. Among different primary sites, patients with GU and Gyn LD tumors had best median survival of 36 months. Among other variables that were examined with respect to their poor prognostic significance, PS $>2(p=0.001)$ and one or fewer number of

(C) Springer Science+Business Media New York 2014

Correspondence to: Prakash Neupane, pneupane@kumc . edu.

Conflict of interest The authors declare that they have no conflict of interests. 
treatment modalities especially in $\operatorname{LD}(p=0.0005)$ were found to be associated significantly with mortality. GI and GU tract tumors were the most common primary sites of disease in our retrospective review. Survival varied according to stage, PS, site of primary disease, use of chemotherapy, and number of treatment modalities used. Further studies are needed to better understand this rare disorder and optimize management approach.

\section{Keywords}

Extrapulmonary; Small cell carcinoma; Limited stage; Extensive stage; Gastrointestinal; Genitourinary; Gynecologic; Head and neck

\section{Introduction}

Extrapulmonary small cell carcinoma (EPSCC) has been a rare and challenging disease entity to manage for over 80 years. Oat cell carcinoma of the mediastinal glands was first described as a distinct clinicopathologic disease entity in 1930-1934 years after small cell carcinoma of the lung had been described - and since that time, its presence has been increasingly recognized [1-3]. Though small cell carcinoma makes up 20-25\% of bronchogenic carcinomas, primary sites outside the lung are much more rare [4]. Many different extrapulmonary sites can be involved, with the GI and GU systems being the most common [1]. Within the GU tract, the bladder and prostate are frequently affected, with the first case of bladder primary described in 1981 [5]. Within the GI tract, these tumors may originate from the oropharynx, salivary glands, parotid glands, colon, rectum, pancreas, or esophagus. Esophageal primary tumors are more often described in Japanese literature [6].

The incidence of EPSCC in North America is estimated to be in the range of 0.1-0.4\%, making it account for approximately 1 in 20 cases of small cell [1,7-9]. Much like pulmonary small cell carcinoma, EPSCC has an aggressive natural history with dissemination typically occurring early and with a high rate of recurrence or relapse [2]. Survival rates in EPSCC are low, with a mean duration of survival for all stages of around 20 months and a 5-year overall survival (OS) as low as $8.1 \%$ [5].

Because of its low incidence, treatment recommendations for EPSCC have originated from case reports, single institution case series, or the extrapolation of results from pulmonary small cell trials $[1,7,9]$. No consensus guidelines exist and further work is needed to improve our understanding of the best way to manage these patients. Multimodality therapy has made a survival improvement in small cell lung cancer, where radiation therapy has been added to traditional chemotherapy regimens resulting in a $5 \%$ improvement in OS and response rates of $50-85 \%$ [10].

We performed this study to review the clinical features, characteristics, and natural disease course of patients at our tertiary referral center over the past 23 years. 


\section{Patients and methods}

\section{Patient selection and data collection}

The study protocol was approved by the Institutional Review Board at The University of Kansas. Medical records for all patients diagnosed with EPSCC over the past 23 years were reviewed. The patients were identified using the University's tumor registry, and an initial list of 42 extrapulmonary patients was created from a total of 321 patients overall who had a small cell carcinoma of any primary site. Pulmonary primary tumors made up 279 of the 321 cases $(87 \%)$. Five patients were excluded from the initial list due to lack of pathologic confirmation of small call histology during chart review, and two were removed due to insufficient information. For the remaining 35 patients, the following data were collected for each patient: age, gender, ECOG performance status (PS), anatomic location, smoking history, documentation of weight loss, number and location of metastatic sites, stage (limited or ED), specific chemotherapy given, surgical or radiation therapy management, use of PCI, and survival information, if available.

\section{Diagnostic workup}

Charts were reviewed to establish documentation of workup for a primary pulmonary etiology when appropriate, including chest X-ray or CT scan. Diagnosis was confirmed by histology, morphology, and/or immunohistochemistry.

\section{Staging}

Patients were excluded if a pulmonary primary site was diagnosed. Patients were classified as LD or ED using the Veteran's Affairs Lung Study Group two-stage system [7, 11]. In this system, tumors are defined as limited stage if confined to a single-radiation therapy treatment port and as extensive stage if they are spread beyond locoregional boundaries [12]. Patients with liver or bone metastasis were staged as having ED.

\section{Statistical analysis}

Kaplan-Meier survival estimates and log-rank tests were used to compare survival between limited and extensive stages of disease and to investigate the association of other possible prognostic factors with survival. SAS ${ }^{\circledR}$ version 9.3 was used for all analysis. An a priori significance level of 0.05 was used to identify significant associations.

\section{Results \\ Patient characteristics}

The patients in the study were predominantly male $(66 \%)$ and had a median age of 68 years (range 25-87 years). Nineteen patients (54\%) had a documented history of tobacco use. ECOG PS was documented for 14 patients, with 11 (79\%) having a PS of 0-1 at the time of diagnosis (Tables 1, 2). 


\section{Primary site of disease}

The anatomic location of the primary disease varied in this series of patients as anticipated. Eleven of the 35 cases ( $31 \%$ ) originated from the GU tract, including the bladder, kidney, ureter, prostate, and urethra. Ten cases (29\%) originated from various locations in the GI tract including the colon, rectum, pancreas, and esophagus. Six cases (17\%) arose from the female reproductive organs, including the cervix, ovary, and Bartholin's glands. Five cases (14\%) were considered head and neck primary tumors from the base of tongue, nasopharynx, maxillary sinus, thyroid, and parotid. Three cases (9\%) were deemed to be from an unknown primary source. Pulmonary primary disease was excluded for all 35 cases including the three with unknown sources (Table 1).

\section{Metastasis and stage}

Fifteen ( $43 \%$ ) patients had metastatic disease at the time of diagnosis, with the liver being the most common site of metastasis ( 9 of 15 cases, $60 \%$ ). Other sites involved in smaller numbers were the brain $(1,7 \%)$, bones $(3,20 \%)$, lung $(1,7 \%)$, and mesentery $(1,7 \%)$. The mean number of sites of metastasis was 1.7 , with only 6 of the 35 cases presenting with $>1$ site of metastasis. Using the VA 2-stage system, 20 cases (57\%) were classified as LD and $15(43 \%)$ were classified ED [11] (Table 2).

\section{Treatment and survival of patients with EPSCC}

Treatment regimens were reviewed to assess whether chemotherapy, surgery, radiation, or prophylactic cranial irradiation were used (Table 3). The results showed 23 of the 35 patients $(66 \%)$ received some form of chemotherapy. The most commonly used chemotherapy regimens included etoposide combined with either cisplatin or carboplatin in 17 (74 \%) of the 23 patients. Other less frequently used chemotherapeutic agents included cyclophosphamide, paclitaxel, gemcitabine, topotecan, doxorubicin, vincristine, and docetaxel.

Surgical excision of the primary tumor was done in 11 of the 35 cases (31\%). Of the cases treated with surgical resection, 9 of the $11(82 \%)$ were staged as LD. Fifteen patients (43\%) were treated with radiation therapy and an additional three patients were treated with prophylactic cranial irradiation with or without definitive radiation. The stage of disease in the patients treated with radiation was nearly equal with 9 LD patients and 7 ED receiving some form of radiation. Interestingly, there were three patients in the cohort who were treated with radiation alone and did not receive chemotherapy or undergo surgical resection.

At the time of this analysis, 9 (26\%) patients were confirmed to be living, with the longest survivor being 13 years removed from diagnosis. All nine of the living patients had been diagnosed with $\mathrm{LD}$, and 8 of the 9 were treated with chemotherapy plus either surgery or radiation.

\section{Prognostic variables}

The median duration of survival for the cohort was 13 months (95\% CI = 7, 36 months). Kaplan-Meier survival curves comparing LD to ED patients reveal a clear survival benefit in favor of $\mathrm{LD}(p<0.0001$, Fig. 1). The median survival for $\mathrm{LD}$ patients was approximately 
36 months, compared to a 5-month median survival for ED patients. Primary site location was also found to be significantly associated with survival $(p=0.01)$. Patients with gynecologic primary sites were all classified as LD and appeared to have the best survival prognosis, with a median survival of 36 months ( $95 \%$ CI lower bound $=14$ months). Similarly, genitourinary primary cases were predominantly classified as LD ( 7 of 11 cases, $64 \%$ ) and had median survival of 36 months (95\%CI lower bound $=2$ months), but the risk of death appeared to be slightly greater earlier on these patients when compared to those with a gynecological diagnosis (Fig. 2). Head and neck patients (3 out of $5 \mathrm{LD}, 60 \%$ ) had a median survival of 17 months ( $95 \%$ CI lower bound $=5$ months) versus 6.5 months for gastrointestinal disease (95\% CI 0.1, 13 months), $60 \%$ (6 of 10) of which were ED. All three cases with unknown primary site diagnoses were classified as ED and had a median survival of 3 months (95\% CI 0.25, 12 months). Other studies on the subject of extrapulmonary small cell cancer have shown a similarly poor survival for patients with a GI primary site [2].

Fewer treatment modalities were found to be highly associated with shortened survival, both in the overall sample $(p=0.0021)$ and within the disease cohorts $(p=0.03$ in $\mathrm{ED}, p=0.03$ in LD) (Fig. 3). In patients with ED, patients with more than one modality had a median survival of 12 months (95\% CI 4, 36 months), compared to 3.5 months (95\% CI 0.1, 7 months) for patients with one or fewer modalities. In LD, the number of treatment modalities was found to be highly associated with survival $(p=0.0005)$. Patients who received at least two modalities had a median survival of 65 months $(95 \% \mathrm{CI}$ lower bound $=$ 14 months), compared to 17 months (95\% CI lower bound = 2 months) for those with one or fewer modalities.

Chemotherapy was found to be associated with prolonged survival, both overall ( $p<0.0001$ and within disease stage ( $p=0.007$ in ED, $p=0.003$ in LD) (Fig. 4). LD patients receiving chemotherapy showed longest median survival at 65 months $(95 \% \mathrm{CI}$ lower bound $=14$ months); LD subjects not receiving chemotherapy survived an average of 7 months (95\% CI lower bound $=2$ months) and were not significantly different from subjects who did receive chemotherapy but had ED (median survival 11 months, $95 \%$ CI 2, 18 months). ED patients not receiving chemotherapy had the shorted median survival at 3 months (95 \% CI $0.1,5$ months).

ECOG PS was also found to be a significant prognostic factor among the 14 patients who had a documented PS in their chart $(p=0.001)$. This result is not surprising, as PS-like the VA's Lung Study Group two-stage system used to stage the disease-is a measure of disease severity. Patients with a good PS ( 0 or 1$)$ were classified more frequently as having LD $(8 / 11,73 \%)$ and had median survival of 35 months, compared to 1 week for those patients with PS of 3 or 4 (Fig. 5).

\section{Discussion}

EPSCC remains a challenging disease to treat and equally challenging to study due to the small number of cases, which prohibits the development of large, randomized clinical trials. Recommendations are often extrapolated from our experience with pulmonary small cell 
cancer, making case series like this one important for furthering our understanding of the optimal management strategies [1]. The disease can involve several primary sites making generalized treatment recommendations even more challenging [4]. In our review of 35 patients, the most common primary sites of disease were the GU and GI tracts at 31 and 29 $\%$, respectively, followed by gynecologic (17\%), head and neck (14\%), and unknown primary $(9 \%)$. A Canadian case series reported primary disease sites comprising of breast (9 $\%)$, GI (20\%), GU (18\%), gynecologic (11\%), head and neck (10\%), thymus (2\%), and unknown primary site (31\%) [12]. The difference in geography, population, and the more number of unknown primary sites in the Canadian series may account for the different frequencies of primary sites when compared to our series. Overall, the GI and GU tracts appear to the most common primary sites. The male-to-female ratio was approximately $2: 1$, which was comparable but slightly higher in favor of males than previously published case series $[1,3,4,12]$.

A unique staging system does not currently exist, but in most reports the VA 2-stage system has been used and the disease is classified as LD or ED. This series included slightly more LD (57\%) patients compared to ED (43\%). This is comparable to the reported incidence of LD $(51 \%)$ versus ED (50\%) in the Canadian series [12]. For patients with ED, the general principal of treatment has involved using systemic chemotherapy with a doublet including a platinum salt and etoposide. Carboplatin and etoposide were the most common regimens described in the studies reviewed, and the most common combination in our series (used in $45 \%$ of patients receiving chemotherapy). The second most common regimen used was cisplatin and etoposide, which was used in $40 \%$ of chemotherapy treatments. Response rates have historically been reported as high as $69 \%$ [13] and $71 \%$ [12]. Radiation was also used in $43 \%$ of the patients in this series. $31 \%$ of patient in the Canadian series received RT [12]. Examination of prognostic factors suggests that patients with more treatment modalities have improved OS, and this is particularly true in ED where those receiving one or fewer have survival of $<1$ month. This case series is limited by its small sample size-as is the case with all studies of this kind-so drawing definitive conclusions about this association is challenging. There is also a clear bias toward healthier, younger patients with better PS being able to tolerate more aggressive therapy, but it seems fair to conclude that for those ED patients who can tolerate combination therapy it should typically be offered.

For LD patients, previous publications have looked at the survival of patients undergoing surgery alone, but the high rate of local or systemic recurrence has frequently led to the addition of adjuvant chemotherapy or radiation, or both. Two patients in our series were treated with surgery alone, with one patient dying at 2 months and the other patient alive at 29 months out from diagnosis. Thirteen of the 20 patients (65\%) received combination therapy with 2 or 3 modalities utilized; $26 \%$ of patients in the Canadian series underwent combined modality treatment [12].

Significant prognostic factors included stage of disease, ECOG PS, site of primary disease, use of chemotherapy, and number of modalities of therapy used, none of which are surprising. However, these results substantiate our prestudy hypothesis. Likewise, the Canadian series found that stage and ECOG PS were significant prognostic factors [12]. However, in the Japanese series chemotherapy (cisplatin based) but not stage of disease was 
reported to be prognostic in a Japanese series of 45 renal EPSCC patients [14]. As anticipated, most of the chemotherapy patients were treated with a small cell lung cancer type of regimen containing etoposide and a platinum. Those patients with a better PS, less metastatic sites of disease, and the ability to undergo a surgical procedure most likely were able to tolerate more extensive treatment; thus, OS is likely confounded by the extent of disease. This is a major limitation of a small case series study such as this one, but designing an adequately powered randomized trial would present significant challenges in such a rare disease entity.

In patients with ED, patients with more than one modality had a median survival of 12 months compared to 3.5 months for patients with one or fewer modalities. In a Canadian series, the median survival for ED was 2 months (range 0.1-108 months) and no statistically significant difference was observed when treated with one or fewer modality vs more than one modality [12]. In LD, patients who received at least two modalities had a median survival of 65 months, compared to 17 months for those with one or fewer modalities. In the Canadian series, the median survival for LD was 34 months (range 0.2-276 months) and CR rates were higher when treated with more than one modality ( $88 \%)$ vs one modality (77\%). However, this did not translate into a statistically significant difference in survival when treated with one or fewer modality vs more than one modality [12]. In a case series comprising of Head \& Neck LD EPSCC patients $(n=12)$ from University of Miami, the median disease-specific survival time was 22 months (95\% CI, 2.6-30) in the radiotherapy or radiotherapy/chemotherapy group $(n=8)$ and was not attained in the other group $(n=4)$. The 1-year disease-specific survival rate was $73 \%$ in the radiotherapy or radiotherapy/ chemotherapy group compared with $67 \%$ in the other group [15].

EPSCC arising from various primary sites exhibits different tumor biology and hence has been associated with different outcomes. Head and neck patients in our series ( 3 out of 5 $\mathrm{LD}, 60 \%$ ) had a median survival of 17 months ( $95 \% \mathrm{CI}$ lower bound $=5$ months). This is comparable to a median survival of 15.2 months reported in a case series of Head \& Neck LD EPSCC patients ( $n=12)$ from University of Miami. Interestingly, patients with parotid cancer $(n=4)$ were found to have a longer median survival of 30 months [15]. The OS was 30 months in one patient with parotid cancer in our series as well. Head and neck patients in the Canadian series ( 9 out of 10 LD, $90 \%$ ) had a relatively longer median survival of 24 months but individual primary site information was not available.

In our series, patients with GI primary site were associated with median survival of 6.5 months and $60 \%$ (6 of 10) of which were ED. The Canadian series reported median survival of 4.4 months in patients with GI primary site and $60 \%$ (13 of 20) were staged as ED [12]. Other studies on the subject of EPSCC have shown a similarly poor survival for patients with a GI primary site $[2,16]$.

Patients with gynecologic primary sites in our series were all classified as LD and appeared to have the best survival prognosis, with a median survival of 36 months. This trend was similar to the Canadian series where patients with gynecologic primary sites $(n=11)$ were all staged as LD and had the best prognosis with median OS of 54.4 months [12]. As 
observed in our series, patients with cervix EPSCC have the most favorable outcome. Previous series have reported a 3-year OS of $60 \%$ with in patients with cervix EPSCC [17].

In our series, one patient with renal EPSCC survived for 10 months, which is comparable to a median survival of 9.9 months (range 6.9-31.6) reported in a Japanese series of 45 renal EPSCC patients [14]. All three cases with unknown primary site diagnoses in our series were classified as ED and had a median survival of 3 months, which is comparable to the reported dismal median survival of 2.5 months in the Canadian series [12].

In summary, EPSCC remains a difficult disease to treat without clearly defined therapy guidelines. In reviewing the literature and examining the experience of our institution in treating these patients, we were able to better define some of the factors that appear to have prognostic significance. When staging patients using the VA 2-stage system, we can begin to recognize trends in how the disease has historically been treated. The therapies provided at our institution were generally in line with those historical methods. For LD patients, we would recommend surgical resection when possible with the addition of adjuvant chemotherapy and or radiation depending on site of disease and likelihood of local recurrence or distant spread. In the ED setting, use of small cell lung cancer regimens of etoposide and a platinum agent in combination with radiation therapy appears to benefit patients over next less-intensive therapy. More data are clearly needed to further our understanding of the optimum management of EPSCC, and our hope is that randomized trials can eventually be developed to more objectively assess the effects of adding the various treatments at our disposal. For now, the management of this entity continues to be a significant challenge for the treating physician with a generally poor prognosis for the patients.

\section{Acknowledgments}

This work was supported by a CTSA grant from NCRR and NCATS awarded to the University of Kansas Medical Center for Frontiers: the Heartland Institute for Clinical and Translational Research \# KL2TR000119. The contents are solely the responsibility of the authors and do not necessarily represent the official views of the NIH, NCRR, or NCATS.

\section{References}

1. Brennan SM. Should extrapulmonary small cell cancer be managed like small cell lung cancer? Cancer. 2010; 116(4):888-895. [PubMed: 20052730]

2. Lee SS. Extrapulmonary small cell carcinoma: single center experience with 61 patients. Acta Oncol. 2007; 46(6):846-851. [PubMed: 17653910]

3. Cicin I. Extrapulmonary small-cell carcinoma compared with small-cell lung carcinoma: a retrospective single-center study. Cancer. 2007; 110(5):1068-1076. [PubMed: 17614337]

4. Kim JH. Extrapulmonary small-cell carcinoma: a single-institution experience. Jpn J Clin Oncol. 2004; 34(5):250-254. [PubMed: 15231859]

5. Choong NWW. Small cell carcinoma of the urinary bladder. Mayo Clinic Experi Cancer. 2005; 103(6):1172-1178.

6. Casas F. Primary small cell carcinoma of the esophagus: a review of the literature with emphasis on therapy and prognosis. Cancer. 1997; 80(8):1366-1372. [PubMed: 9338459]

7. Soto DE. Limited-stage extrapulmonary small cell carcinoma: outcomes after modern chemotherapy and radiotherapy. Cancer J (Sudbury, Mass). 2007; 13(4):243-246. 
8. Cicin I. Extrapulmonary small cell carcinoma localized in lymph nodes: is it a different clinical entity? Acta Oncol. 2009; 48(3):354-360. [PubMed: 18979286]

9. van der Heijden HFM. Extrapulmonary small cell carcinoma. Southern medical journal (Birmingham, Ala). 2005; 98(3):345-349.

10. Pignon JP. A meta-analysis of thoracic radiotherapy for smallcell lung cancer. New England $\mathbf{J}$ Med. 1992; 327(23):1618-1624. [PubMed: 1331787]

11. Clark R. Small-cell lung cancer: treatment progress and prospects. Oncology (Williston Park, NY). 1998; 12(5):647-658. discussion 61-63.

12. Haider K. Extrapulmonary small cell cancer: a Canadian province's experience. Cancer. 2006; 107(9):2262-2269. [PubMed: 16998932]

13. Lo Re G. Extrapulmonary small cell carcinoma: a single-institution experience and review of the literature. Ann Oncol. 1994; 5(10):909-913. [PubMed: 7696162]

14. Lee SY, Hsu HH, Lin HY, Chen YC, Wong YC, Wang LJ, et al. Factors associated with the survival of patients with primary small cell carcinoma of the kidney. Int J Clin Oncol. 2013; 18(1): 139-147. [PubMed: 22138976]

15. Hatoum GF, Patton B, Takita C, Abdel-Wahab M, LaFave K, Weed D, et al. Small cell carcinoma of the head and neck: the university of Miami experience. Int J Radiat Oncol Biol Phys. 2009; 74(2):477-481. [PubMed: 19004574]

16. Brenner B, Tang LH, Klimstra DS, Kelsen DP. Small-cell carcinomas of the gastrointestinal tract: a review. Int J Clin Oncol. 2004; 22(13):2730-2739.

17. Hoskins PJ, Swenerton KD, Pike JA, Lim P, Aquino-Parsons C, Wong F, et al. Small-cell carcinoma of the cervix: fourteen years of experience at a single institution using a combinedmodality regimen of involved-field irradiation and platinum-based combination chemotherapy. Int J Clin Oncol. 2003; 21(18):3495-3501. 


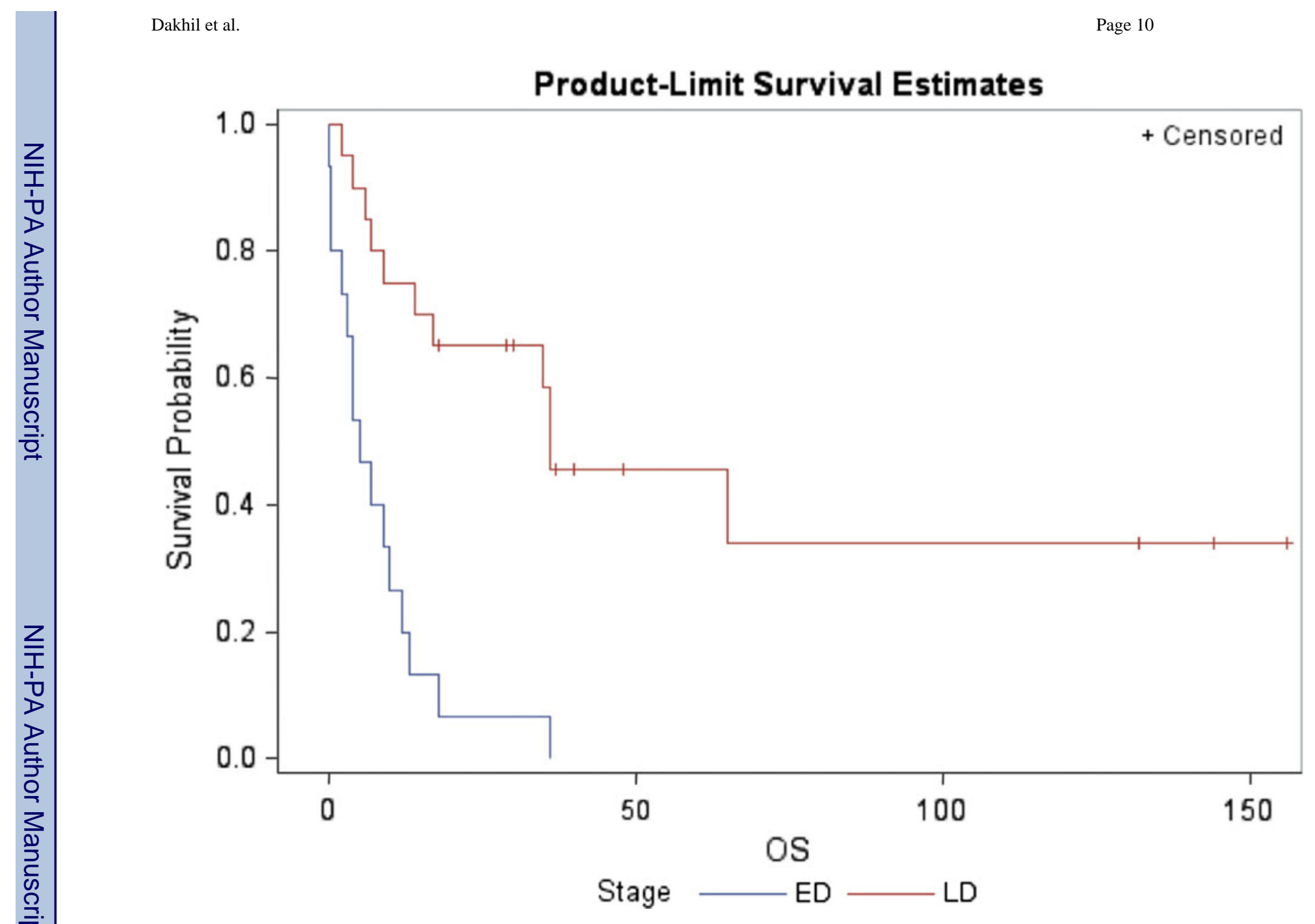

Fig. 1.

Kaplan-Meier survival curves for limited ( $r e d$ ) and extensive (blue) disease 


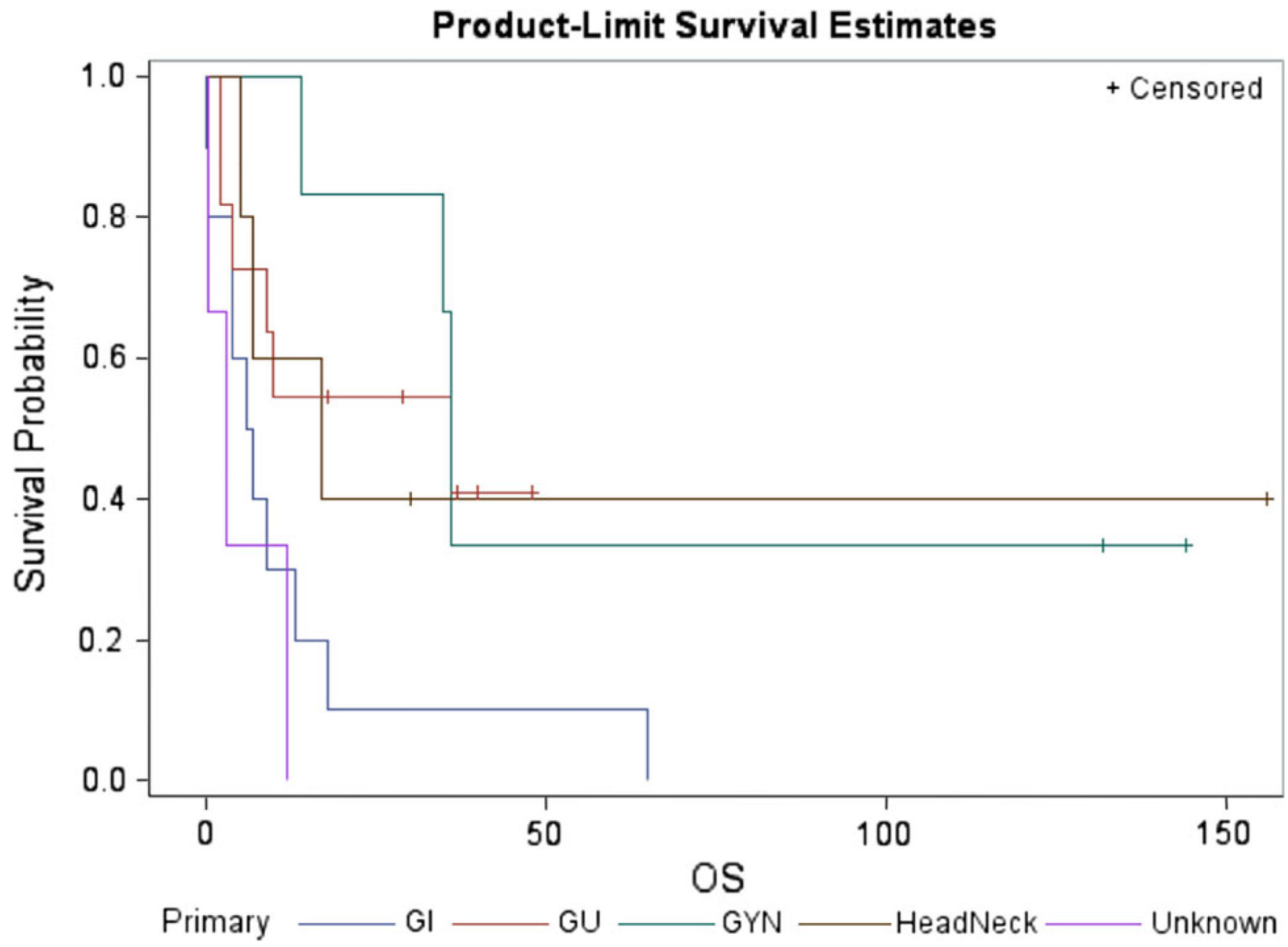

Fig. 2.

Kaplan-Meier survival curves by primary disease site 


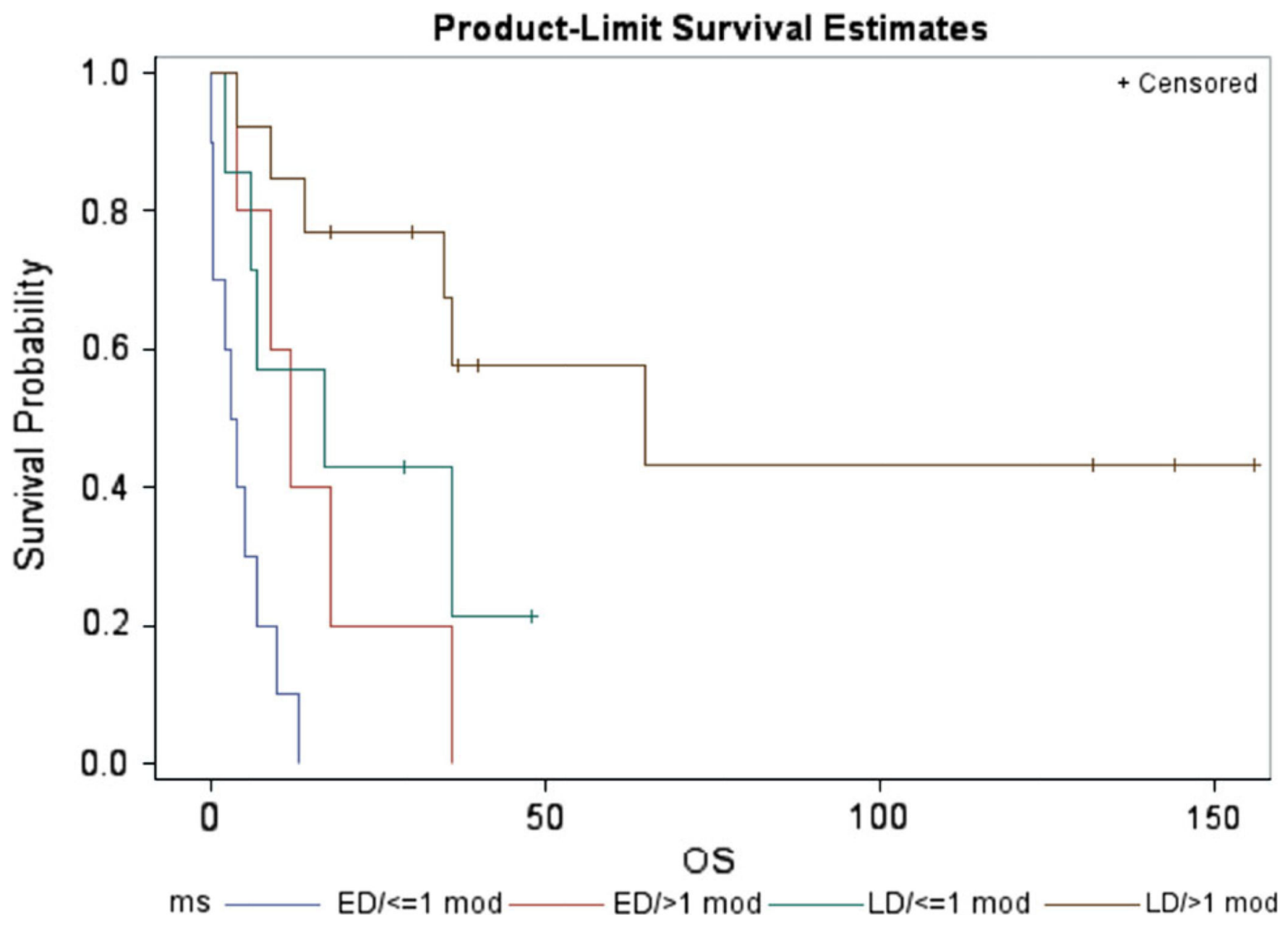

Fig. 3.

Kaplan-Meier survival curves by disease stage and number of treatment modalities 


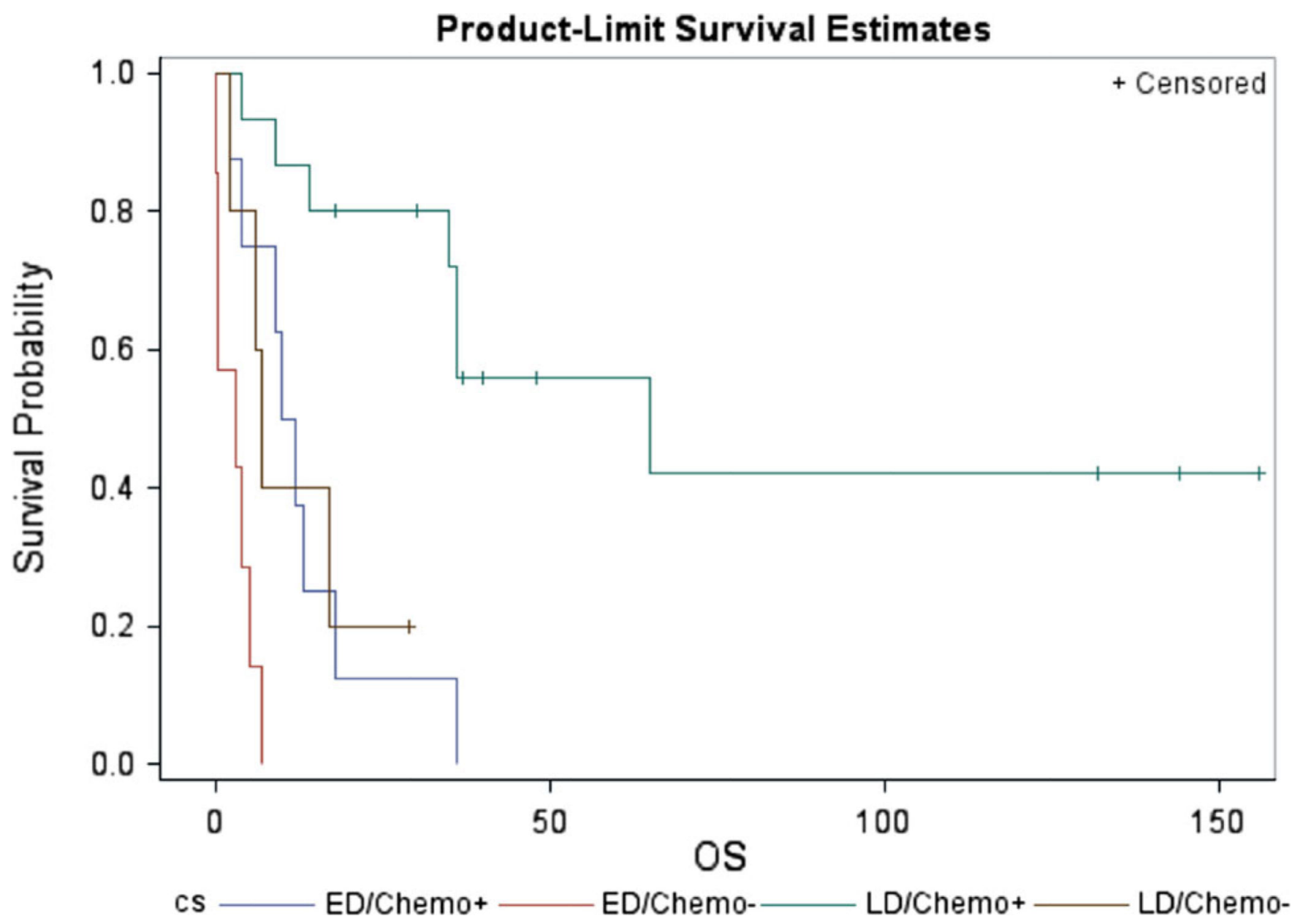

Fig. 4.

Kaplan-Meier survival curves by disease stage and chemotherapy treatment 


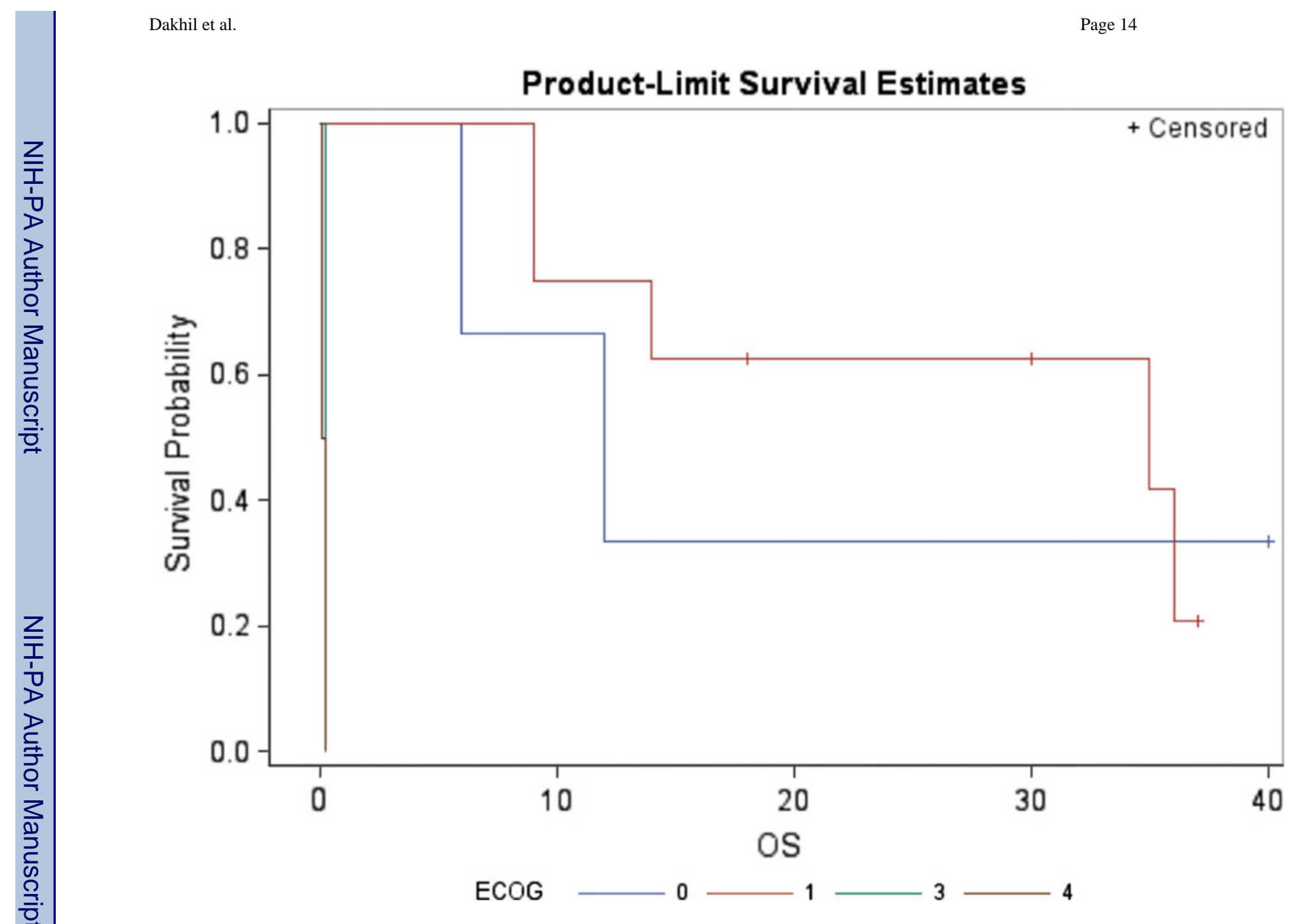

Fig. 5.

Kaplan-Meier survival curves by ECOG performances status 


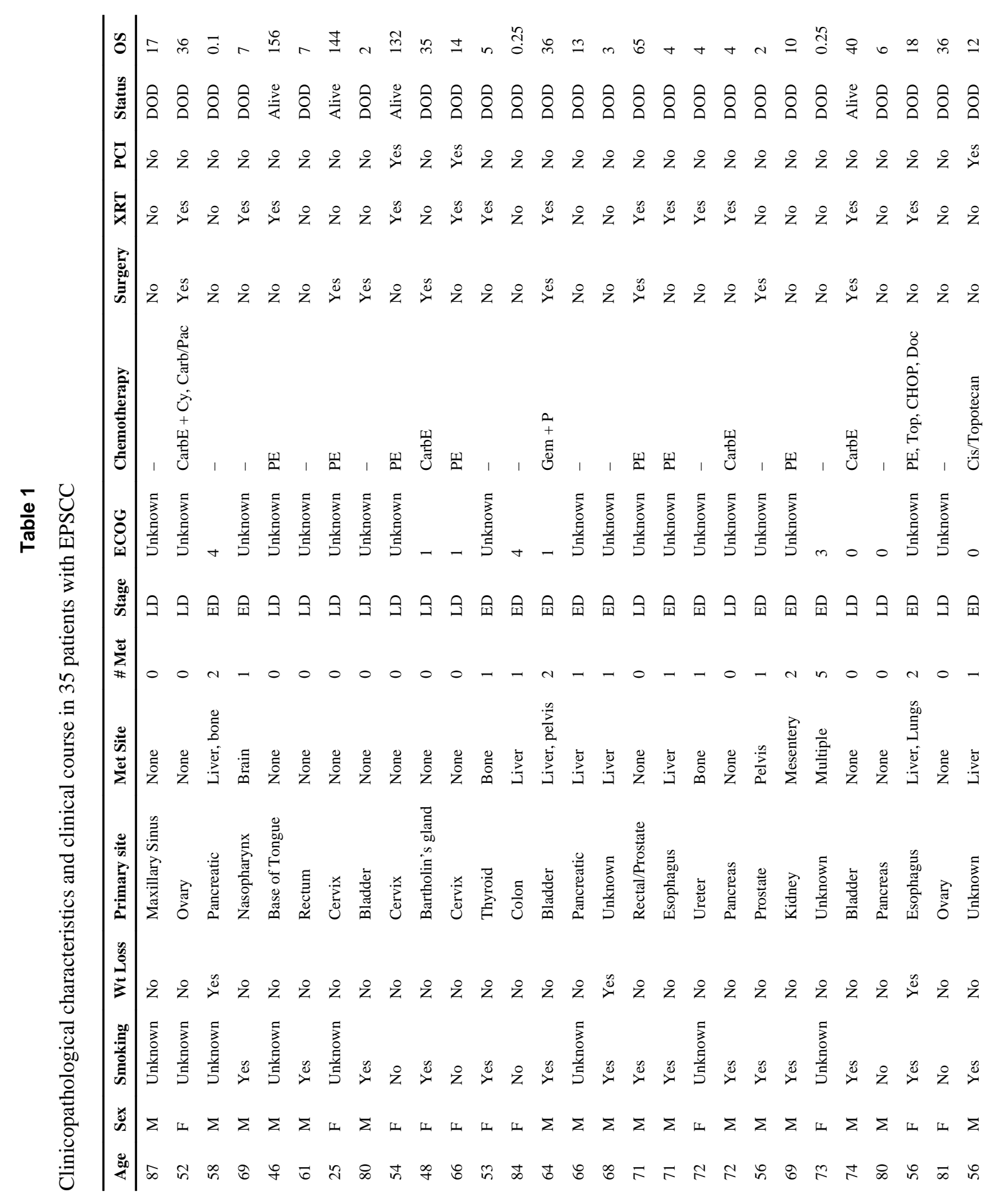

Med Oncol. Author manuscript; available in PMC 2015 October 01. 
Table 2

Demographic and clinical characteristics

\begin{tabular}{llll}
\hline & $\begin{array}{l}\text { Limited disease } \\
(\boldsymbol{n}=\mathbf{2 0})\end{array}$ & $\begin{array}{l}\text { Extensive } \\
\text { disease }(\boldsymbol{n}=\mathbf{1 5})\end{array}$ & $\begin{array}{l}\text { Overall } \\
(\boldsymbol{n}=\mathbf{3 5})\end{array}$ \\
\hline $\begin{array}{l}\text { Age (years) }{ }^{a} \\
\text { Male }\end{array}$ & $71.5(53,79.5)$ & $66(56,71)$ & $68(54,74)$ \\
ECOG PS & $13(65 \%)$ & $10(67 \%)$ & $23(66 \%)$ \\
0 & $2(25 \%)$ & $1(7 \%)$ & $3(9 \%)$ \\
1 & $6(75 \%)$ & $2(13 \%)$ & $8(23 \%)$ \\
2 & $0(0 \%)$ & $0(0 \%)$ & $0(0 \%)$ \\
3 & $0(0 \%)$ & $1(7 \%)$ & $1(3 \%)$ \\
4 & $0(0 \%)$ & $2(13 \%)$ & $2(6 \%)$ \\
Missing & $12(60 \%)$ & $9(60 \%)$ & $21(60 \%)$ \\
Weight loss & & & $19(54 \%)$ \\
Smoking history & $10(50 \%)$ & $9(60 \%)$ & $8(23 \%)$ \\
Missing & $4(20 \%)$ & $4(27 \%)$ & $11(31 \%)$ \\
Surgery & $9(45 \%)$ & $2(13 \%)$ & $15(43 \%)$ \\
Radiotherapy & $13(65 \%)$ & $6(40 \%)$ & $23(66 \%)$ \\
Chemotherapy & $15(75 \%)$ & $8(53 \%)$ & $18(51 \%)$ \\
$>1$ Modalities & $13(65 \%)$ & $5(33 \%)$ & $26 \%$ \\
Survival rate & $45 \%$ & $0 \%$ & $13(7,36)$ \\
Overall survival (months) & $a$ & $5(0.25,10)$ & \\
\hline & $36(9,36)$ & &
\end{tabular}

${ }^{a}$ Median (25th, 75th percentiles) 


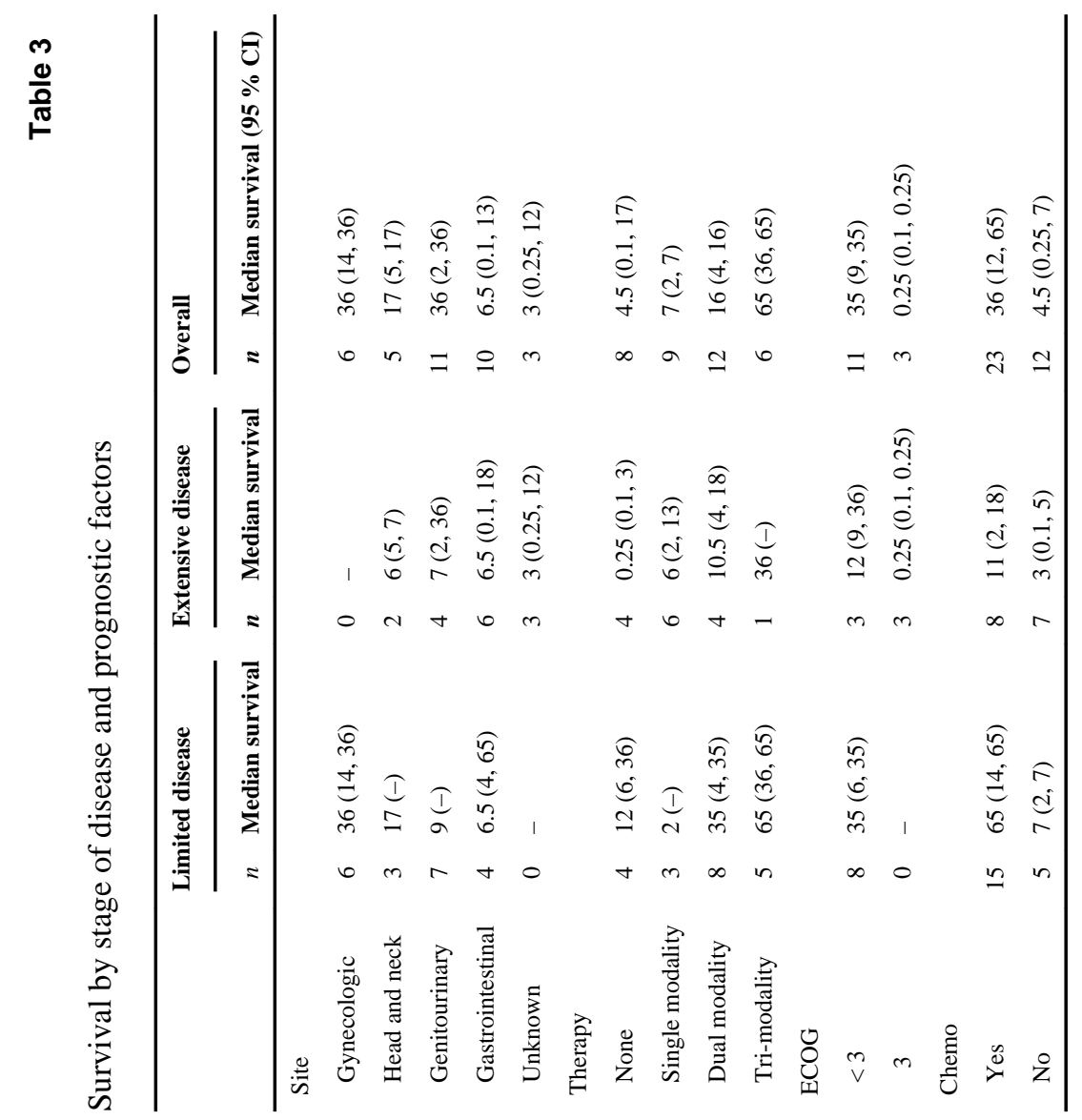

